\title{
Reactivity to UV Radiation Exposure Monitoring Using Personal Exposure Devices for Skin Cancer Prevention: Longitudinal Observational Study
}

Bridget G Parsons ${ }^{1}$, MSPH; Elizabeth S Nagelhout ${ }^{2}$, MPH, PhD; Ali P Wankier ${ }^{1}$, BS; Nan Hu ${ }^{3}$, PhD; Riley Lensink ${ }^{1}$, BA; Angela Zhu ${ }^{1}$, BS; Katy Nottingham ${ }^{1}$, BS; Douglas Grossman ${ }^{1}$, MD, PhD; Jakob D Jensen ${ }^{4}$, PhD; Yelena P Wu ${ }^{1,5}$, $\mathrm{PhD}$

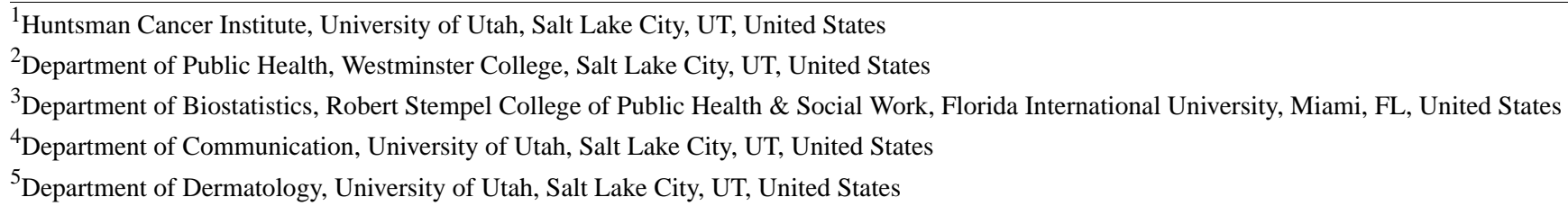

\section{Corresponding Author:}

Yelena P Wu, PhD

Huntsman Cancer Institute

University of Utah

2000 Circle of Hope Drive

Salt Lake City, UT, 84112

United States

Phone: 18012135653

Email: yelena.wu@utah.edu

\section{Abstract}

Background: Emerging UV radiation (UVR) monitoring devices may present an opportunity to integrate such technology into skin cancer prevention interventions. However, little is known about the effects of using a wearable UVR monitor on adults' and children's sun protection-related behaviors and attitudes (eg, cancer worry and perceived risk). Understanding the potential role of reactivity and seasonal effects will help inform the use of objective monitors in the context of skin cancer prevention research, including intervention studies.

Objective: The aim of this study is to examine the potential reactivity associated with a wearable personal UVR monitor, specifically the effects associated with reported sun-protective behaviors and skin cancer-related attitudes, which are often the targets of skin cancer preventive interventions.

Methods: Child-parent dyads ( $\mathrm{n}=97$ dyads) were asked to wear a UVR monitoring device during waking hours for 2 weeks. Participants were asked to sync the device daily with a smartphone app that stored the UVR exposure data. Participants were blinded to their UVR exposure data during the 2-week period; thus, the smartphone app provided no feedback to the participants on their UVR exposure. Participants completed self-report questionnaires assessing sun-protective behaviors, sunburn, tanning, skin self-examination, skin cancer-related knowledge, perceived risk, cancer worry, response efficacy, and intentions to change behaviors over the 2-week period. Linear regressions were conducted to investigate changes in the outcomes over time and to account for the role of the season of study participation.

Results: Regression results revealed that there was a significant decrease over time for several sun protection outcomes in children, including time spent outdoors on weekends $(P=.02)$ and weekdays $(P=.008)$, sunscreen use $(P=.03)$, reapplication $(P<.001)$, and unintentional tanning $(P<.001)$. There were no significant changes over time in children's and parents' UVR exposure, sunburn occurrence, or sun protection attitudes. Season of participation was associated with several outcomes, including lower sunscreen use $(P<.001)$, reapplication $(P<.001)$, sunburns $(P=.01)$, intentions to change sun-protective behaviors $(P=.02)$, and intentional $(P=.008)$ and unintentional tanning $(P=.01)$ for participants who participated in the fall versus the summer.

Conclusions: The findings from this study suggest that daily use of a UVR monitoring device over a 2-week period may result in changes in certain sun-protective behaviors. These results highlight the importance of identifying and addressing potential reactivity to UVR monitoring devices, especially in the context of skin cancer preventive intervention research. Ultimately, 
objectively assessed UVR exposure could be integrated into the outcome assessment for future testing of skin cancer prevention interventions.

(JMIR Mhealth Uhealth 2021;9(9):e29694) doi: 10.2196/29694

\section{KEYWORDS}

ultraviolet radiation exposure; wearable device; melanoma; melanoma prevention; mHealth; digital health; eHealth; UVR monitoring; mobile phone

\section{Introduction}

\section{Background}

Mobile or personal health monitors are frequently used by the general public and in the context of health behavior research [1]. For example, FitBit, one of the most popular wearable activity monitors, reported 2 million new users in 2019, bringing its total active users to 29.6 million [2]. In the past decade, $>300$ studies using FitBit as an outcome measure were registered with the National Institutes of Health [3]. Although physical activity monitors are frequently used in research settings, monitors related to environmental exposures and their associated health behaviors are in the early phases of development, testing, and dissemination [4-6]. In the context of skin cancer prevention, assessment of an individual's UV radiation (UVR) exposure is essential and is becoming more feasible given the availability of personal UVR monitors.

Melanoma is the deadliest type of skin cancer and the fifth most common form of cancer [7]. UVR exposure from the sun or artificial sources is the primary modifiable risk factor for melanoma [8]. Efforts to prevent melanoma focus on decreasing individuals' UVR exposure through sun-protective behaviors, such as wearing sunscreen and clothing that covers the skin, and avoiding peak UVR hours, which are typically assessed through self-report measures [9]. There has been a growing number of objective UVR assessment methods that can quantify personal UVR exposure and be useful for documenting the efficacy of melanoma preventive interventions $[4,10]$. However, a potential concern with using objective UVR exposure assessments is reactivity or the possibility that simply monitoring a behavior may in and of itself result in behavior change $[11,12]$. For example, it is possible that individuals who are aware that their UVR exposure is being monitored could choose to use sun-protection methods more consistently. For example, in the physical activity assessment literature, it has been found that after individuals use objective assessments of their exercise behaviors, changes can be seen in both their behaviors and exercise-related attitudes (eg, covering more distance and reporting great perceived exertion) [13]. In addition, it is not known to what extent personal UVR exposure based on objective assessments could differ depending on environmental factors such as the season. For instance, individuals may make different sun-protection choices based on seasonal differences in weather and temperature [14]. Studies have found that individuals who rely on ambient temperature as a method for determining the need for sun protection, particularly in the winter $[15,16]$, are more likely to receive sunburns in cool weather [17] and tend to use clothing such as long sleeves and pants for warmth rather than sun protection [14].

Although prior studies of objective UVR exposure monitors have not yet examined potential reactivity effects, this phenomenon has been more fully explored in physical activity research [18]. In the literature, there are mixed findings regarding whether monitoring behavior alone is associated with behavior change. Most studies reporting on behavioral changes among adults and children related to monitoring used devices such as pedometers that provided feedback to the participants on physical activity outcomes (eg, number of steps taken daily) [19-22]. In the studies of monitors that did not provide feedback, most found that monitoring alone was not associated with behavior change [23-26].

\section{Objective}

In relation to melanoma prevention, studies have examined the effects of providing periodic feedback to adults, adolescents, and children on UVR exposure; information on the risk of sunburn; and advice about sun protection methods [27-29]. For example, the provision of UVR exposure feedback is associated with less UVR exposure on weekends and an increase in the use of some forms of sun protection [27-29]. Other studies have examined variability in UVR exposure and sun-protective behaviors among different populations, such as rural and urban children, and periods, such as during vacation [30-34]. However, it remains unknown to what extent UVR measurement itself, in the absence of providing feedback on UVR exposure, is associated with changes in sun-protective behaviors and attitudes (eg, cancer worry and perceived risk) in adults and children. Some sun-protection behaviors (eg, sunscreen use, hat use, and wearing pants) are not directly measured by the device. Our hypothesis, based on the literature on physical activity and limited UVR exposure reactivity, is that wearing a UVR monitoring device could lead individuals to become more aware of their UVR exposure and, as a result, change their behaviors and attitudes related to sun exposure. For example, in the physical activity literature, attitudes related to exercise such as perceived efficiency, intensity of effort, and fatigue have been found to increase with accelerometer use [13]. We hypothesize that sun-protection behaviors increase over the monitoring period and that UVR measured by the device would decrease as a result of increased UVR exposure awareness. Understanding the potential role of reactivity and seasonal effects will help inform the use of objective monitors in the context of skin cancer prevention research, including intervention studies. This pilot study was designed to validate the use of a UVR monitoring device in larger skin cancer prevention interventions designed for parents and children [5]. The goal of this study is to assess the potential reactivity associated with UVR exposure 
monitoring among adults and children as it relates to sun-protective behaviors and skin cancer prevention-related attitudes, which are often the targets of skin cancer preventive interventions [35-37].

\section{Methods}

\section{Participants}

Potential participants were recruited through health and community events (eg, health fairs and farmers' markets) and invitation letters that were mailed to residents with a child aged between of 8 and 17 years. A web-based marketing resource was used to obtain the addresses of potential participants living in Utah [38]. To be eligible to participate in this study, adults who were residents of Utah, aged $\geq 18$ years, had at least one child aged between 8 and 17 years, had a smartphone with Bluetooth and Wi-Fi capabilities, were willing to use a smartphone app that shared their UVR exposure information with the research team, did not have a pacemaker (because of potential interference from the UVR monitor), and were able to read and write in English were included. For children to be eligible to participate, they had to be aged between 8 and 17 years, live with a primary caregiver in Utah, have no previous melanoma diagnosis, and not have a pacemaker. In total, 224 adults expressed interest in participating in the study. Of the 224 adults, $150(67.0 \%)$ were screened for eligibility. Of the 150 adults screened, $116(77.3 \%)$ were screened as eligible and $34(22.7 \%)$ as ineligible. Reasons for ineligibility included not having a child in the desired age range $(28 / 34,82 \%)$, not having a smartphone with the necessary specifications $(5 / 34,15 \%)$, and not being able to read or write in English (1/34, 3\%). A total of 97 parents (77\% biological mothers) and 97 children (mean age 12.7 years, SD 2.7) were enrolled between June 2018 and October 2018. These months were selected because of the high ambient UVR levels in the region during those months [39]. All study procedures were approved by the relevant institutional review board.

\section{Procedures}

Participants were asked to wear a UVR monitoring device (Shade wearable UVR sensor, model V1.00, YouV Labs Inc) $[4,40]$ on their clothing for a 2 -week period. They completed the baseline assessment before being given the UVR monitoring devices to wear. A monitoring period of 2 weeks was selected to allow the capture of data from both weekdays and weekends for $>1$ week (to minimize missing data for either weekdays or weekend days). Participants were asked to synchronize the Shade device daily with a smartphone app that stored the UVR exposure data in a cloud-based server accessed by the research team. Participants were blinded to their UVR exposure data during the 2-week period; thus, the smartphone app provided no feedback to the participants on their UVR exposure. Changes in UVR exposure were analyzed by comparing total UVR exposure in the first week of study participation with total UVR exposure in the second week of study participation. Participants were asked to complete electronically delivered questionnaires via REDCap (Research Electronic Data Capture) [41] at the beginning and end of the 2-week period about sun-protective behaviors - such as wearing sunscreen and protective clothing (10 items) [42], number of sunburns received (one item) [42], tanning (two items) [43], skin self-examination (one item) [44], skin cancer-related knowledge (10 items) [45]—and relevant attitudes such as cancer worry (four items) [46], perceived risk (two items) [47], efficacy of skin cancer preventive behaviors (four items) [48], and intentions to change preventive behaviors (five items) [49-51]. Parents reported on these constructs for both themselves and their child, and children provided self-reports. All items except knowledge (true or false items) were asked on a 5-point Likert scale. The multi-item scales, including skin cancer-related knowledge, cancer worry $(\alpha=.87$, child report; $\alpha=.90$, parent report; parents not asked to report on child), perceived risk ( $\alpha=.75$, child report; $\alpha=.82$, parent report; $\alpha=.84$, parent report on child), efficacy of preventive behaviors ( $\alpha=.89$, child report; $\alpha=.94$, parent report; $\alpha=.95$, parent report on child), and intention to change preventive behaviors $(\alpha=.39$, child report; $\alpha=.55$, parent report; $\alpha=.66$, parent report on child), were summed. After completing their study participation, each participant was provided with a gift card and a report summarizing their UVR exposure over the 2-week period.

\section{Analyses}

Descriptive statistics were used to summarize the participants' demographic information and summary statistics for the outcomes of interest. Linear regressions were used to examine potential changes over the 2-week period in UVR exposure measured from the device, sun-protective behaviors, sunburn, tanning, skin self-examination, and in summed scales for skin cancer-related knowledge, perceived risk, cancer worry, response efficacy, and intentions to change behaviors. Additional linear regressions were conducted with seasonal (summer [June-August] vs fall [September-October]) assessment time points (baseline vs exit) and their interactions as predictors and the same outcomes as dependent variables [52]. Season was included in these models based on the existing literature [53], and it was significantly related to multiple outcomes in this study.

\section{Results}

\section{Sample Characteristics}

In total, 97 parent-child dyads participated in the study. Of the 97 parents, $73(77 \%)$ were biological mothers, $83(87 \%)$ were non-Hispanic White, and $5(5 \%)$ were Hispanic. Of the 97 children (mean age 12.7 years, SD 2.7), 81 (85\%) were non-Hispanic White, 8 (8\%) were Hispanic, and $56(59 \%)$ were female (Table 1). 
Table 1. Demographic characteristics of participating parents and children $(\mathrm{N}=97)^{\mathrm{a}}$.

\begin{tabular}{|c|c|c|}
\hline Characteristics & Parents & Children \\
\hline Age (years), mean (SD) & $41.6(6.3)$ & $12.7(2.7)$ \\
\hline Gender (female), n (\%) & $73(77)$ & $56(59)$ \\
\hline \multicolumn{3}{|l|}{ Race, $\mathbf{n}(\%)$} \\
\hline Non-Hispanic White & $83(87)$ & $81(85)$ \\
\hline Hispanic & $5(5)$ & $8(8)$ \\
\hline Asian or Asian American & $5(5)$ & $4(4)$ \\
\hline Other & $2(2)$ & $2(2)$ \\
\hline \multicolumn{3}{|l|}{ Fitzpatrick skin type (I-VI) } \\
\hline Type I & $9(9)$ & $2(2)$ \\
\hline Type II & $15(15)$ & $17(17)$ \\
\hline Type III & $42(43)$ & $41(42)$ \\
\hline Type IV & $24(25)$ & $32(33)$ \\
\hline Type V & $5(5)$ & $3(3)$ \\
\hline \multicolumn{3}{|l|}{ Marital status, n (\%) } \\
\hline Married or marriage-like relationship & $84(88)$ & $\mathrm{N} / \mathrm{A}^{\mathrm{b}}$ \\
\hline Divorced/separated & $9(10)$ & N/A \\
\hline Widowed & $2(2)$ & N/A \\
\hline \multicolumn{3}{|l|}{ Level of education, $n$ (\%) } \\
\hline High school graduate or GED ${ }^{c}$ & $8(8)$ & N/A \\
\hline Some college, including 2-year degree & $41(42)$ & N/A \\
\hline Bachelor's degree & $25(26)$ & N/A \\
\hline Master's/doctoral degree & $21(22)$ & N/A \\
\hline \multicolumn{3}{|l|}{ Household income (US \$), n (\%) } \\
\hline$<50,000$ & $23(24)$ & N/A \\
\hline$>50,000$ & $64(67)$ & N/A \\
\hline Would prefer not to report & $8(8)$ & N/A \\
\hline
\end{tabular}

${ }^{\mathrm{a}}$ Two families did not complete the baseline questionnaire, thus each of the categories have a total of 95 .

${ }^{b} \mathrm{~N} / \mathrm{A}$ : not applicable (children were not asked this question).

${ }^{\mathrm{c}}$ GED: General Education Development.

\section{Descriptive Statistics}

Descriptive statistics (means and SDs) for UVR exposure, sun-protective and risk behaviors, and skin cancer-related attitudes are presented in Table 2. 
Table 2. Descriptive statistics for UV radiation exposure and sun-protective behaviors, knowledge, and attitudes.

\begin{tabular}{|c|c|c|c|c|c|c|}
\hline & \multicolumn{2}{|l|}{ Child self-report } & \multicolumn{2}{|l|}{ Parent report on child } & \multicolumn{2}{|l|}{ Parent self-report } \\
\hline & Baseline, mean (SD) & Exit, mean (SD) & Baseline, mean (SD) & Exit, mean (SD) & Baseline, mean (SD) & Exit, mean (SD) \\
\hline $\mathrm{UVR}^{\mathrm{a}}$ exposure $^{\mathrm{b}}$ & $6.00(6.09)$ & $4.76(5.27)$ & $N / A^{c}$ & N/A & $6.89(6.38)$ & $6.11(6.62)$ \\
\hline UVR exposure (adjusted) $^{\mathrm{d}}$ & $6.47(5.94)$ & $5.50(5.07)$ & N/A & N/A & $7.56(6.58)$ & $6.87(6.81)$ \\
\hline Hours outdoors: weekday & $2.69(1.75)$ & $2.24(1.65)$ & $2.72(1.47)$ & $2.09(1.43)$ & $2.79(1.80)$ & $2.01(1.68)$ \\
\hline Hours outdoors: weekend & $3.22(2.09)$ & $2.49(1.57)$ & $3.55(1.97)$ & $2.76(1.68)$ & $3.88(1.91)$ & $2.72(1.66)$ \\
\hline Sunscreen $^{\mathrm{e}}$ & $2.35(1.45)$ & $1.83(1.13)$ & $2.52(1.39)$ & $2.02(1.21)$ & $2.53(1.45)$ & $2.11(1.33)$ \\
\hline Reapplication $^{\mathrm{e}}$ & $1.95(1.15)$ & $1.50(0.84)$ & $1.98(1.19)$ & $1.60(0.86)$ & $1.87(1.19)$ & $1.60(0.96)$ \\
\hline Long sleeves ${ }^{\mathrm{e}}$ & $1.76(0.93)$ & $2.12(1.03)$ & $1.86(1.00)$ & $2.16(1.04)$ & $1.87(1.06)$ & $2.20(1.15)$ \\
\hline Pants $^{\mathrm{e}}$ & $2.76(1.21)$ & $3.03(1.36)$ & $2.52(1.19)$ & $2.82(1.32)$ & $3.02(1.34)$ & $3.19(1.37)$ \\
\hline $\mathrm{Hat}^{\mathrm{e}}$ & $1.31(0.82)$ & $1.37(0.91)$ & $1.37(0.73)$ & $1.37(0.90)$ & $1.97(1.33)$ & $1.84(1.07)$ \\
\hline Shade $\mathrm{e}^{\mathrm{e}}$ & $2.56(1.04)$ & $2.51(1.06)$ & $2.45(0.96)$ & $2.43(1.01)$ & $2.67(1.00)$ & $2.55(1.02)$ \\
\hline Avoid peak hours ${ }^{\mathrm{e}}$ & $2.08(0.94)$ & $2.14(1.02)$ & $2.33(1.19)$ & $2.34(1.04)$ & $2.55(1.14)$ & $2.35(1.00)$ \\
\hline Sunglasses $^{\mathrm{e}}$ & $1.92(1.18)$ & $1.59(1.01)$ & $1.89(1.18)$ & $1.60(0.99)$ & $3.58(1.40)$ & $3.19(1.34)$ \\
\hline Sunburn & $0.23(0.57)$ & $0.16(0.43)$ & $0.15(0.36)$ & $0.16(0.42)$ & $0.10(0.30)$ & $0.18(0.44)$ \\
\hline Intentional tanning ${ }^{\mathrm{e}}$ & $1.24(0.63)$ & $1.15(0.47)$ & $1.16(0.45)$ & $1.09(0.33)$ & $1.13(0.48)$ & $1.08(0.31)$ \\
\hline Unintentional tanning $^{\mathrm{e}}$ & $2.88(1.17)$ & $1.80(1.07)$ & $2.95(1.33)$ & $1.80(1.02)$ & $2.93(1.16)$ & $1.90(1.05)$ \\
\hline Indoor tanning ${ }^{\mathrm{e}}$ & $1.00(0)$ & $1.00(0)$ & $1.00(0)$ & $1.00(0)$ & $1.00(0)$ & $1.00(0)$ \\
\hline Skin self-examination ${ }^{\mathrm{e}}$ & $1.88(0.32)$ & $1.88(0.32)$ & $0.09(0.29)$ & $0.17(0.38)$ & $0.16(0.37)$ & $0.12(0.33)$ \\
\hline Skin cancer knowledge $\mathrm{f}^{\mathrm{f}}$ & $2.98(1.42)$ & $3.23(1.49)$ & N/A & $\mathrm{N} / \mathrm{A}$ & $4.08(1.01)$ & $4.24(0.95)$ \\
\hline UVR knowledge $^{\mathrm{f}}$ & $2.97(1.27)$ & $3.31(1.13)$ & N/A & $\mathrm{N} / \mathrm{A}$ & $3.84(0.67)$ & $3.88(0.65)$ \\
\hline Total knowledge $\mathrm{e}^{\mathrm{f}}$ & $5.94(2.35)$ & $6.55(2.25)$ & N/A & $\mathrm{N} / \mathrm{A}$ & $7.92(1.29)$ & $8.11(1.22)$ \\
\hline Perceived risk ${ }^{\mathrm{g}}$ & $4.52(1.68)$ & $4.48(1.79)$ & $5.67(1.75)$ & $5.83(1.60)$ & $6.33(1.88)$ & $6.19(1.83)$ \\
\hline Cancer worry $^{\mathrm{h}}$ & $7.36(3.34)$ & $7.10(3.20)$ & N/A & N/A & $9.52(3.73)$ & $9.12(3.52)$ \\
\hline Response efficacy ${ }^{i}$ & $15.29(3.44)$ & $15.40(3.81)$ & $16.53(3.84)$ & $17.3(2.74)$ & $17.00(3.57)$ & $17.37(2.89)$ \\
\hline Intentions to change $\mathrm{e}^{\mathrm{j}}$ & $10.66(3.93)$ & $10.92(4.53)$ & $11.35(4.73)$ & $11.74(4.89)$ & $13.66(5.04)$ & $13.38(4.96)$ \\
\hline
\end{tabular}

${ }^{a} U V R:$ UV radiation.

${ }^{\mathrm{b}}$ First 7 days versus last 7 days of the 2-week wearing period.

${ }^{\mathrm{c}} \mathrm{N} / \mathrm{A}$ : not applicable (parents not asked to report this on children).

${ }^{d}$ First 7 days versus last 7 days of the 2-week wearing period adjusted to include participants that had at least 4 days of UVR data.

${ }^{\mathrm{e}}$ Response options included never $=1$ to always $=5$.

${ }^{\mathrm{f}} 0=$ false, $1=$ true. Possible range of 0-5, or 0-10 for total knowledge.

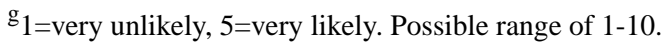

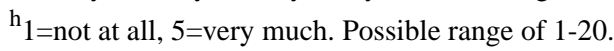

${ }^{\mathrm{i}} 1=$ strongly disagree, $5=$ strongly agree. Possible range of 1-20.

$\mathrm{j}_{1}=$ no and I do not intend to start doing so in the next 6 months, 5=yes, I have been for more than 6 months. Possible range of 1-25.

\section{Regression Analyses}

Regression results revealed that there was a significant change over time for several outcomes (Tables 3-5). In addition, separate analyses examining the relationship between season alone and these outcomes are provided in the table footnotes. 
Table 3. Child self-report regression models examining association between time and season of participation and sun-protective behaviors ${ }^{\mathrm{a}}$.

\begin{tabular}{|c|c|c|c|c|c|c|c|c|c|c|c|c|}
\hline \multirow[t]{2}{*}{ Outcome } & \multirow[t]{2}{*}{$R^{2}$} & \multirow[t]{2}{*}{$F$ test $(d f)$} & \multicolumn{5}{|l|}{ Time } & \multicolumn{5}{|c|}{ Season } \\
\hline & & & $\beta$ & SE (B) & $\mathrm{B}$ & $t$ test & $P$ value & $\beta$ & $\mathrm{SE}(ß)$ & $\mathrm{B}$ & $t$ test & $P$ value \\
\hline $\mathrm{UVR}^{\mathrm{b}}$ exposure ${ }^{\mathrm{c}}$ & 0.00 & $0.17(181)$ & 0 & 1.17 & 0.00 & 0 & .99 & -.05 & 1.29 & -0.64 & -0.49 & .62 \\
\hline UVR exposure adjusted $^{\mathrm{d}}$ & 0.01 & $0.38(137)$ & -.06 & 1.26 & -0.70 & -0.56 & .58 & -.04 & 1.30 & -0.42 & -0.33 & .75 \\
\hline Hours outdoors: weekday & 0.02 & $1.36(181)$ & -.17 & 0.33 & -0.59 & -1.77 & .08 & -.11 & 0.36 & -0.39 & -1.06 & .29 \\
\hline Hours outdoors: weekend & 0.04 & $2.59(181)$ & -.16 & 0.37 & -0.61 & -1.67 & .09 & .01 & 0.39 & 0.02 & 0.04 & .96 \\
\hline Sunscreen & 0.29 & $23.62(181)$ & -.22 & 0.22 & -0.59 & -2.69 & .008 & -.56 & 0.24 & -1.52 & -6.39 & $<.001$ \\
\hline Reapplication & 0.18 & $13.10(181)$ & -.28 & 0.18 & -0.57 & -3.12 & .002 & -.43 & 0.20 & -0.92 & -4.60 & $<.001$ \\
\hline Long sleeves & 0.06 & $3.83(181)$ & .07 & 0.19 & 0.14 & 0.76 & .45 & -.01 & 0.20 & -0.01 & -0.06 & .95 \\
\hline Pants & 0.05 & $3.29(181)$ & .08 & 0.24 & 0.19 & 0.81 & .42 & .18 & 0.26 & 0.45 & 1.74 & .08 \\
\hline Hat & 0.02 & $1.48(181)$ & .01 & 0.17 & 0.02 & 0.11 & .92 & -.15 & 0.18 & -0.27 & -1.46 & .15 \\
\hline Shade & 0.05 & $3.22(181)$ & .08 & 0.20 & 0.17 & 0.88 & .34 & -.11 & 0.22 & -0.23 & -1.09 & .28 \\
\hline Avoid peak hours & 0.07 & $4.63(181)$ & .17 & 0.18 & 0.34 & 1.89 & .06 & -.02 & 0.20 & -0.03 & -0.16 & .87 \\
\hline Sunglasses & 0.06 & $3.64(181)$ & -.14 & 0.21 & -0.32 & -1.52 & .13 & -.14 & 0.23 & -0.32 & -1.41 & .16 \\
\hline Sunburn & 0.09 & $5.64(181)$ & -.12 & 0.09 & -0.12 & -1.29 & .20 & -.36 & 0.10 & -0.36 & -3.65 & $<.001$ \\
\hline Intentional tanning & 0.04 & $2.43(181)$ & -.09 & 0.11 & -0.10 & -0.96 & .34 & -.18 & 0.11 & -0.20 & -1.75 & .08 \\
\hline Unintentional tanning & 0.18 & $13.42(181)$ & -.43 & 0.22 & -1.06 & -4.88 & $<.001$ & -.12 & 0.24 & -0.27 & -1.15 & .25 \\
\hline Indoor tanning & $-^{\mathrm{e}}$ & - & - & - & - & - & - & - & - & - & - & - \\
\hline Skin self-exam & 0.01 & $0.70(181)$ & .05 & 0.06 & 0.03 & 0.50 & .62 & .13 & 0.07 & 0.09 & 1.26 & .21 \\
\hline Skin cancer knowledge & 0.01 & $0.68(181)$ & .03 & 0.28 & 0.09 & 0.33 & .75 & .01 & 0.31 & 0.03 & 0.09 & .93 \\
\hline UVR knowledge & 0.02 & $1.21(181)$ & .12 & 0.24 & 0.30 & 1.25 & .39 & .09 & 0.26 & 0.22 & 0.85 & .39 \\
\hline Total knowledge & 0.02 & $1.13(181)$ & .08 & 0.45 & 0.39 & 0.87 & .39 & .05 & 0.49 & 0.25 & 0.51 & .61 \\
\hline Perceived risk & 0.01 & $0.07(181)$ & -.03 & 0.34 & -0.03 & -0.11 & .92 & .01 & 0.37 & 0.01 & 0.01 & .99 \\
\hline Cancer worry & 0.01 & $0.79(181)$ & -.08 & 0.63 & -0.52 & -0.82 & .41 & -.11 & 0.69 & -0.76 & -1.09 & .27 \\
\hline Response efficacy & 0.01 & $0.35(181)$ & .01 & 0.69 & 0.01 & 0.01 & .99 & -.07 & 0.75 & -0.54 & -0.72 & .48 \\
\hline Intentions to change & 0.09 & $5.50(181)$ & .06 & 0.78 & 0.46 & 0.59 & .56 & -.22 & 0.85 & -1.89 & -2.23 & .03 \\
\hline
\end{tabular}

${ }^{a}$ Models examining the relationship between season alone and the outcomes of interest indicated that shade seeking, avoidance of peak hours, wearing sunglasses, and intentional tanning were lower in the fall versus summer, whereas pants and hat wearing were higher in the fall.

${ }^{\mathrm{b}} \mathrm{UVR}$ : UV radiation.

${ }^{\mathrm{c}}$ First 7 days versus last 7 days of the 2-week wearing period.

${ }^{\mathrm{d}}$ First 7 days versus last 7 days of the 2-week wearing period adjusted to include participants who had at least 4 days of UVR data.

${ }^{\mathrm{e}}$ No indoor tanning was reported. 
Table 4. Parent report on child regression models examining the association between time and season of participation and sun-protective behaviors ${ }^{\mathrm{a}}$.

\begin{tabular}{|c|c|c|c|c|c|c|c|c|c|c|c|c|}
\hline \multirow[t]{2}{*}{ Outcome } & \multirow[t]{2}{*}{$R^{2}$} & \multirow[t]{2}{*}{$F$ test (df) } & \multicolumn{5}{|l|}{ Time } & \multicolumn{5}{|c|}{ Season } \\
\hline & & & $\beta$ & SE (B) & $\mathrm{B}$ & $t$ test & $P$ value & $\beta$ & SE (ß) & $\mathrm{B}$ & $t$ test & $P$ value \\
\hline Hours outdoors: weekday & 0.08 & $5.16(183)$ & -.22 & 0.28 & -0.68 & -2.42 & .02 & -.16 & 0.31 & -0.48 & 1.56 & .12 \\
\hline Hours outdoors: weekend & 0.07 & $4.30(183)$ & -.25 & 0.35 & -0.94 & -2.68 & .008 & -.14 & 0.38 & -0.55 & -1.44 & .15 \\
\hline Sunscreen & 0.23 & $18.26(183)$ & -.18 & 0.23 & -0.51 & 2.17 & .03 & -.41 & 0.26 & -1.16 & -4.53 & $<.001$ \\
\hline Reapplication & 0.20 & $14.86(183)$ & -.29 & 0.21 & -0.69 & -3.31 & $<.001$ & -.47 & 0.23 & -1.15 & -5.02 & $<.001$ \\
\hline Long sleeves & 0.04 & $2.29(183)$ & -.02 & 0.21 & 0.03 & 0.16 & .87 & -.10 & 0.23 & -0.24 & -1.02 & .31 \\
\hline Pants & 0.04 & $2.47(183)$ & -.01 & 0.24 & -0.03 & -0.11 & .91 & -.05 & 0.28 & -0.13 & -0.49 & .62 \\
\hline Hat & 0.01 & $0.23(183)$ & -.02 & 0.17 & 0.04 & -0.24 & .81 & .03 & 0.19 & -0.06 & -0.33 & .74 \\
\hline Shade & 0.08 & $5.19(183)$ & .04 & 0.20 & 0.09 & 0.45 & .66 & -.19 & 0.22 & -0.41 & -1.86 & .07 \\
\hline Avoid peak hours & 0.03 & $1.97(183)$ & .01 & 0.24 & 0.03 & 0.11 & .91 & -.12 & 0.26 & -0.31 & 1.17 & .25 \\
\hline Sunglasses & 0.02 & $1.33(183)$ & -.14 & 0.24 & -0.33 & -1.42 & .16 & -.07 & 0.26 & -0.18 & -0.68 & .50 \\
\hline Sunburn & 0.06 & $3.57(183)$ & -.01 & -0.07 & -0.01 & -0.02 & .98 & -.26 & 0.08 & -0.20 & -2.53 & .01 \\
\hline Intentional tanning & 0.05 & $3.35(183)$ & -.17 & 0.09 & -0.18 & -1.86 & .07 & -.27 & 0.11 & -0.29 & -2.67 & .008 \\
\hline Unintentional tanning & 0.24 & $18.58(182)$ & -.48 & 0.23 & -1.30 & -5.72 & $<.001$ & -.24 & 0.25 & 0.66 & -2.62 & .01 \\
\hline Indoor tanning & $-^{b}$ & - & - & - & - & - & - & - & - & - & - & - \\
\hline Skin self-exam & 0.01 & $0.75(183)$ & -.12 & 0.07 & -0.08 & -1.27 & .21 & -.03 & 0.07 & -0.02 & -0.25 & .81 \\
\hline Perceived risk & 0.03 & $2.04(183)$ & .10 & 0.32 & 0.33 & 1.04 & .30 & .23 & 0.35 & 0.76 & 2.20 & .03 \\
\hline Response efficacy & 0.02 & $0.94(183)$ & .16 & 0.64 & 1.05 & 1.65 & .10 & .07 & 0.70 & 0.48 & 0.69 & .49 \\
\hline Intentions to change & 0.07 & $4.42(183)$ & .05 & 0.89 & 0.43 & 0.48 & .63 & -.24 & 0.97 & -2.35 & -2.42 & .02 \\
\hline
\end{tabular}

${ }^{a}$ Models examining the relationship between season alone and the outcomes of interest indicated that shade seeking, avoidance of peak hours, and hours spent outside on weekdays were lower in the fall versus summer, whereas pants wearing was higher in the fall.

${ }^{\mathrm{b}}$ No indoor tanning was reported. 
Table 5. Parent self-report regression models examining the association between time and season of participation and sun-protective behaviors ${ }^{\mathrm{a}}$.

\begin{tabular}{|c|c|c|c|c|c|c|c|c|c|c|c|c|}
\hline \multirow[t]{2}{*}{ Outcome } & \multirow[t]{2}{*}{$R^{2}$} & \multirow[t]{2}{*}{$F$ test $(d f)$} & & \multicolumn{5}{|c|}{ Season } \\
\hline & & & B & $\mathrm{SE}(ß)$ & $\mathrm{B}$ & $t$ test & $P$ value & B & $\mathrm{SE}(ß)$ & $\mathrm{B}$ & $t$ test & $P$ value \\
\hline $\mathrm{UVR}^{\mathrm{b}}$ exposure $^{\mathrm{c}}$ & 0.02 & $0.92(179)$ & -.08 & 1.24 & -1.05 & -0.85 & .40 & -.14 & 1.38 & -1.85 & -1.34 & .18 \\
\hline UVR exposure adjusted $^{\mathrm{d}}$ & 0.01 & $0.64(162)$ & -.08 & 1.30 & -1.05 & -0.81 & .42 & -.14 & 1.45 & -1.87 & -1.29 & .20 \\
\hline Hours outdoors: weekday & 0.01 & $0.32(177)$ & .01 & 0.33 & 0.01 & 0.01 & .99 & -.07 & 0.36 & -0.25 & -0.70 & .49 \\
\hline Hours outdoors: weekend & 0.02 & $0.98(183)$ & .04 & 0.33 & 0.14 & 0.42 & .67 & -.06 & 0.36 & -0.19 & -0.54 & .59 \\
\hline Sunscreen & 0.16 & $11.44(183)$ & -.16 & 0.25 & -0.44 & -1.79 & .08 & -.40 & 0.27 & -1.13 & -4.21 & $<.001$ \\
\hline Reapplication & 0.14 & $9.91(183)$ & -.14 & 0.19 & -0.31 & -1.55 & .12 & -.36 & 0.22 & -0.82 & -3.78 & $<.001$ \\
\hline Long sleeves & 0.04 & $2.28(183)$ & .04 & 0.21 & 0.09 & 0.43 & .67 & -.09 & 0.23 & -0.20 & -0.87 & .39 \\
\hline Pants & 0.06 & $3.32(183)$ & -.02 & 0.25 & -0.05 & -0.18 & .86 & .10 & 0.28 & 0.26 & 0.95 & .35 \\
\hline Shade & 0.08 & $5.43(183)$ & .01 & 0.18 & 0.02 & 0.13 & .89 & -.16 & 0.20 & -0.33 & -1.61 & .11 \\
\hline Avoid peak hours & 0.05 & $2.99(183)$ & .04 & 0.20 & 0.08 & 0.39 & .70 & -.01 & 0.22 & -0.03 & -0.12 & .91 \\
\hline Sunglasses & 0.02 & $1.37(183)$ & -.16 & 0.26 & -0.44 & -1.68 & .09 & -.03 & 0.29 & -0.08 & -0.27 & .79 \\
\hline Sunburn & 0.03 & $2.03(183)$ & .16 & 0.07 & 0.12 & 1.71 & .09 & -.06 & 0.08 & -0.04 & -0.56 & .58 \\
\hline Intentional tanning & 0.01 & $0.58(183)$ & -.08 & 0.08 & -0.06 & -0.83 & .41 & -.09 & 0.08 & -0.08 & -0.94 & .35 \\
\hline Unintentional tanning & 0.22 & $16.52(183)$ & -.37 & 0.20 & -0.88 & -4.33 & $<.001$ & -.14 & 0.22 & -0.35 & -1.57 & .12 \\
\hline Indoor tanning & $\underline{\mathrm{e}}^{\mathrm{e}}$ & - & - & - & - & - & - & - & - & - & - & - \\
\hline Skin self-exam & 0.01 & $0.88(183)$ & .04 & 0.07 & 0.03 & 0.45 & .65 & .03 & 0.07 & 0.02 & 0.24 & .81 \\
\hline Skin cancer knowledge & 0.01 & $0.26(183)$ & .04 & 0.19 & 0.09 & 0.46 & .65 & -.03 & 0.21 & -0.06 & -0.29 & .77 \\
\hline Total knowledge & 0.01 & $0.27(183)$ & .04 & 0.24 & 0.09 & 0.40 & .69 & -.05 & 0.26 & -0.12 & -0.47 & .64 \\
\hline Perceived risk & 0.01 & $0.84(183)$ & -.03 & 0.35 & -0.12 & -0.34 & .73 & .12 & 0.39 & 0.45 & 1.15 & .25 \\
\hline Cancer worry & 0.02 & $0.91(183)$ & -.05 & 0.70 & -0.36 & -0.52 & .60 & .12 & 0.77 & 0.87 & 1.13 & .26 \\
\hline Response efficacy & 0.01 & $0.33(183)$ & .06 & 0.62 & 0.41 & 0.66 & .51 & -.03 & 0.68 & -0.20 & -0.30 & .77 \\
\hline Intentions to change & 0.02 & $2.01(183)$ & .03 & 0.95 & 0.26 & 0.27 & .79 & -.07 & 1.05 & -0.68 & -0.65 & .52 \\
\hline
\end{tabular}

${ }^{a}$ Models examining the relationship between season alone and the outcomes of interest indicated that shade seeking, unintentional, and intentional tanning were lower in the fall versus summer.

${ }^{\mathrm{b}} \mathrm{UVR}$ : UV radiation.

${ }^{\mathrm{c}}$ First 7 days versus last 7 days of the two-week wearing period.

${ }^{\mathrm{d}}$ First 7 days versus last 7 days of the two-week wearing period adjusted to include participants that had at least 4 days of UVR data.

${ }^{\mathrm{e}}$ No indoor tanning was reported.

There was a significant decrease in children's sunscreen use based on child $\left(F_{3,178}=23.62 ; P<.001 ; R^{2}=0.29\right)$ and parent report $\left(F_{3,180}=18.21 ; P<.001 ; R^{2}=0.23\right)$. When season was held constant, sunscreen use decreased in children over the 2-week study period based on child $\left(\beta=-.22 ; t_{3}=-2.69 ; P=.008\right)$ and parent report $\left(\beta=-.18 ; t_{3}=-2.17 ; P=.03\right)$. There were also decreases in reapplication of sunscreen in children based on child $\left(\beta=-.28 ; t_{3}=-3.12 ; P=.002\right)$ and parent report $(\beta=-.69$; $\left.t_{3}=-3.31 ; P<.001\right)$. There was a significant decrease in reported unintentional tanning for children based on child report $(\beta=-.43$; $\left.t_{3}=-4.88 ; P<.001\right)$ and parent report $\left(\beta=-.48 ; t_{3}=-5.72 ; P<.001\right)$ and parents $\left(~\left(=-.37 ; t_{3}=-4.33 ; P<.001\right)\right.$. In addition, there were decreases in the hours children spent outside on weekdays
$\left(B=-.22 ; t_{3}=-2.42 ; P=.02\right)$ and weekends $\left(B=-.25 ; t_{3}=-2.68\right.$; $P=.008)$ based on parent report. There were no significant changes over time in UVR exposure, sunburn, or attitudes.

Season of participation was associated with several outcomes. Reported sunscreen use was lower for children based on child $\left(\beta=-.56 ; t_{3}=-6.39 ; P<.001\right)$ and parent report $\left(\beta=-.41 ; t_{3}=-4.53\right.$; $P<.001)$ and parents $\left(\beta=-.40 ; t_{3}=-4.21 ; \quad P<.001\right)$ who participated in fall versus summer. Reapplication of sunscreen was also lower for children based on child $\left(B=-.43 ; t_{3}=-4.60\right.$; $P<.001)$ and parent report $\left(B=-.47 ; t_{3}=-5.02 ; P<.001\right)$ and parents $\left(\beta=-.36 ; t_{3}=-3.78 ; P<.001\right)$ who participated in fall. Reported child sunburns per child $\left(B=-.36 ; t_{3}=-3.65 ; P<.001\right)$ and parent report $\left(B=-.26 ; t_{3}=-2.53 ; P=.01\right)$ and intentions to 
change were lower for children who participated in the fall $\left(\beta=-.22 ; t_{3}=-2.23 ; P=.03\right)$ than in the summer $\left(B=-.24 ; t_{3}=-2.42\right.$; $P=.02)$. Intentional tanning $\left(\beta=-.27 ; t_{3}=-2.67 ; P=.008\right)$ and unintentional tanning $\left(B=-.24 ; t_{3}=-2.62 ; P=.01\right)$ were lower for children who participated in the fall based on parent report. Perceived risk for cancer was higher for children who participated in the fall compared with those who participated in summer based on parent report $\left(\beta=.23 ; t_{3}=2.20 ; P=.03\right)$.

\section{Discussion}

\section{Principal Findings}

The current findings indicate that it may be important to identify and address the reactivity to UVR monitoring devices among children and parents. Our results provide initial evidence that the use of a UVR monitoring device may be associated with changes in sun-protective behaviors. In the context of intervention studies seeking to improve the use of sun-protection behaviors, the reactivity effects observed, particularly the decreased use of sun-protection behaviors, could potentially dampen the detection of desired intervention effects.

In contrast to most previous studies that found that monitoring alone did not lead to behavior change, some significant changes in skin cancer preventive behaviors over time were detected, including in children's time spent outside on weekdays and weekends, use and reapplication of sunscreen, and unintentional tanning. Notably, almost all of the changes in outcomes observed were decreases in reported sun-protective behaviors over time. This could indicate that wearing the UVR monitoring device initially led participants to increase their use of sun-protective behaviors, and then over time, the effect wore off as participants acclimatized to wearing the device. This has been observed in the physical activity literature, for example, that the first measurement day was the most active day for participants, and this tapered off in subsequent days [20]. Future studies could further explore the role of reactivity associated with UVR monitoring devices by assessing participants over a longer wearing period and incorporating an initial familiarization phase to allow for any reactivity effects to subside. In addition, future studies could consider systematically excluding the first few days of UVR monitoring data during analysis.
We observed seasonal differences in sun-protection behaviors based on both child reports and parent reports, which is consistent with the findings of prior studies that individuals may make different sun-protection choices based on weather or time of year [14-17]. Our findings confirm the importance of controlling for seasonal effects, either through statistical methods or study design. For example, studies could take place during the course of a single season (eg, summer).

This study has several strengths and limitations. Strengths include that our study is one of the first to examine the reactivity to UVR exposure monitoring using a wearable UVR monitoring device. In addition, parents and children were included in this study, and they could both benefit from skin cancer interventions that incorporate wearable UVR monitoring devices. Limitations include a study sample from a single location, which may limit generalizability to populations in other geographic areas. Future studies could include a control group that does not wear a sensor to disentangle the potential contributions of the self-report method of assessment of sun-protection behaviors and other outcomes. Future studies could further assess potential reactivity associated with the use of wearable UVR monitoring devices that do provide feedback on exposure and account for other factors that may impact potential reactivity, such as age, amount of time spent outdoors, and geographic location.

\section{Conclusions}

This study is among the first to assess the potential reactivity associated with UVR exposure monitoring. Reactivity effects should be further examined in both intervention and observational contexts to better understand the impact of UVR monitoring on sun-protective behaviors and other relevant clinical outcomes. Ultimately, objectively assessed UVR exposure is an important measure to be integrated into outcome assessment for future testing of skin cancer prevention interventions. In the context of intervention testing, researchers who deploy objective UVR measures may want to compare intervention outcomes between individuals who used a UVR monitor and those who did not. In addition, when examining intervention effects on objectively assessed UVR exposure, researchers may want to analyze UVR data with and without the first few days of UVR data collected to minimize potential reactivity effects.

\section{Acknowledgments}

This work was supported by Dave Overholt, the National Cancer Institute of the National Institutes of Health (K07CA196985 to YPW; 3DP2EB022360-01S1 to JDJ), a Pilot Project Award from the American Cancer Society Huntsman Cancer Institute Institutional Research Grant (129785-IRG-16-190-01-IRG), and the Office of Communications, Genetic Counseling Shared Resource, and Cancer Biostatistics Shared Resource supported by grant P30CA042014 to Huntsman Cancer Institute. Data for this project were collected using REDCap (Research Electronic Data Capture), which is supported by the National Institutes of Health (8UL1TR000105, formerly UL1RR025764). The content is solely the responsibility of the authors and does not necessarily represent the official views of the National Institutes of Health. The authors greatly appreciate Peter Kaplan and Emmanuel Dumont for their guidance on and assistance with using the Shade device and Jared Luther for assistance with data management. The authors are grateful for the study recruitment efforts of Mark Hyde, Garrett Harding, Jennyffer Morales, and Jane Ostler. The authors also thank James Carrington for his assistance in organizing the results.

\section{Conflicts of Interest}

None declared. 


\section{References}

1. Feehan LM, Geldman J, Sayre EC, Park C, Ezzat AM, Yoo JY, et al. Accuracy of Fitbit Devices: Systematic Review and Narrative Syntheses of Quantitative Data. JMIR Mhealth Uhealth 2018 Aug 09;6(8):e10527 [FREE Full text] [doi: 10.2196/10527] [Medline: 30093371]

2. Fitbit reports 2019 fourth quarter and full year results. FitBit, Inc. 2020. URL: https://investor.fitbit.com/press-releases/ press-release-details/2020/Fitbit-Reports-2019-Fourth-Quarter-and-Full-Year-Results/default.aspx [accessed 2021-03-22]

3. US National Library of Medicine. ClinicalTrials.gov. 2020. URL: https://clinicaltrials.gov/ [accessed 2021-02-12]

4. Banerjee S, Hoch E, Kaplan P, Dumont E. A comparative study of wearable ultraviolet radiometers. In: Proceedings of the IEEE Life Sciences Conference (LSC). 2017 Presented at: IEEE Life Sciences Conference (LSC); Dec. 13-15, 2017; Sydney, NSW, Australia. [doi: 10.1109/lsc.2017.8268131]

5. Nagelhout ES, Lensink R, Zhu A, Parsons BG, Jensen JD, Wu YP. The feasibility and acceptability of using a wearable uv radiation exposure monitoring device in adults and children: cross-sectional questionnaire study. JMIR Dermatol 2020 Apr 29;3(1):e15711. [doi: 10.2196/15711] [Medline: PMC7546527]

6. Cai S, Xu X, Yang W, Chen J, Fang X. Materials and designs for wearable photodetectors. Adv Mater 2019 Feb 20;31(18):1808138. [doi: 10.1002/adma.201808138] [Medline: $\underline{\text { 30785644] }}$

7. Cancer stat facts: Melanoma of the skin. NIH Surveillance Epidemiology and End Results Program. 2020. URL: https:/ /seer.cancer.gov/statfacts/html/melan.html [accessed 2020-08-27]

8. Armstrong BK, Kricker A. How much melanoma is caused by sun exposure? Melanoma Res 1993;3(6):395-401. [doi: 10.1097/00008390-199311000-00002] [Medline: 8161879]

9. Hillhouse J, Turrisi R, Jaccard J, Robinson J. Accuracy of self-reported sun exposure and sun protection behavior. Prev Sci 2012 Oct 27;13(5):519-531 [FREE Full text] [doi: 10.1007/s11121-012-0278-1] [Medline: 22855253]

10. Glanz K, Mayer JA. Reducing ultraviolet radiation exposure to prevent skin cancer methodology and measurement. Am J Prev Med 2005 Aug;29(2):131-142. [doi: 10.1016/j.amepre.2005.04.007] [Medline: 16005810]

11. Baumann S, Groß S, Voigt L, Ullrich A, Weymar F, Schwaneberg T, et al. Pitfalls in accelerometer-based measurement of physical activity: The presence of reactivity in an adult population. Scand J Med Sci Sports 2017 Oct 12;28(3):1056-1063. [doi: 10.1111/sms.12977] [Medline: 28921747]

12. Motl RW, McAuley E, Dlugonski D. Reactivity in baseline accelerometer data from a physical activity behavioral intervention. Health Psychol 2012;31(2):172-175. [doi: 10.1037/a0025965] [Medline: 22023436]

13. Vandoni M, Correale L, Del Bianco M, Marin L, Codrons E. Does reactivity to accelerometers occur in a single trial? Brief report in a sample of young adults. J Health Psychol 2016 Feb 15;22(11):1458-1462. [doi: 10.1177/1359105316628758] [Medline: 26880758]

14. Andersen PA, Buller DB, Walkosz BJ, Scott MD, Beck L, Liu X, et al. Environmental variables associated with vacationers' sun protection at warm weather resorts in North America. Environ Res 2016 Apr;146:200-206. [doi: 10.1016/j.envres.2015.12.034] [Medline: 26775001]

15. van der Leun JC, Piacentini RD, de Gruijl FR. Climate change and human skin cancer. Photochem Photobiol Sci 2008;7(6):730. [doi: 10.1039/b719302e] [Medline: 18528559]

16. Andersen PA, Buller DB, Walkosz BJ, Scott MD, Maloy JA, Cutter GR, et al. Environmental cues to UV radiation and personal sun protection in outdoor winter recreation. Arch Dermatol 2010 Nov 15;146(11):200-206. [doi: 10.1001/archdermatol.2010.327] [Medline: 21079060]

17. Hill D, White V, Marks R, Theobald T, Borland R, Roy C. Melanoma prevention: Behavioral and nonbehavioral factors in sunburn among an Australian urban population. Preven Med 1992 Sep;21(5):654-669. [doi: 10.1016/0091-7435(92)90072-p] [Medline: 1438112]

18. Silfee VJ, Haughton CF, Jake-Schoffman DE, Lopez-Cepero A, May CN, Sreedhara M, et al. Objective measurement of physical activity outcomes in lifestyle interventions among adults: A systematic review. Preventive Medicine Reports 2018 Sep;11:74-80. [doi: 10.1016/j.pmedr.2018.05.003] [Medline: 29984142]

19. Clemes SA, Matchett N, Wane SL. Reactivity: an issue for short-term pedometer studies? Br J Sports Med 2007 Jun 05;42(1):68-70. [doi: 10.1136/bjsm.2007.038521] [Medline: 18178685]

20. Dössegger A, Ruch N, Jimmy G, Braun-Fahrländer C, Mäder U, Hänggi J. Reactivity to accelerometer measurement of children and adolescents. Med Sci Sports Exerc 2014;46(6):1140. [doi: 10.1249/mss.0000000000000215] [Medline: 24219978]

21. Bravata DM, Smith-Spangler C, Sundaram V, Gienger AL, Lin N, Lewis R, et al. Using pedometers to increase physical activity and improve health. J Am Med Assoc 2007 Nov 21;298(19):2296. [doi: 10.1001/jama.298.19.2296] [Medline: $\underline{18029834]}$

22. Foley JT, Beets MW, Cardinal BJ. Monitoring children's physical activity with pedometers: reactivity revisited. J Exer Sci Fit 2011 Dec;9(2):82-86. [doi: 10.1016/s1728-869x(12)60002-1]

23. Lubans DR, Morgan PJ, Tudor-Locke C. A systematic review of studies using pedometers to promote physical activity among youth. Prev Med 2009 Apr;48(4):307-315. [doi: 10.1016/j.ypmed.2009.02.014] [Medline: 19249328] 
24. Vincent S, Pangrazi R. Does reactivity exist in children when measuring activity levels with pedometers? Pediatr Exerc Sci 2002;14(1):56-63. [doi: 10.1123/pes.14.1.56]

25. Matevey C, Rogers LQ, Dawson E, Tudor-Locke C. Lack of reactivity during pedometer self-monitoring in adults. Meas Phys Educ Exerc Sci 2006 Mar;10(1):1-11. [doi: 10.1207/s15327841mpee1001_1]

26. Behrens TK, Dinger MK. Motion sensor reactivity in physically active young adults. Res Q Exerc Sport 2007 Mar;78(2):1-8. [doi: 10.1080/02701367.2007.10599397] [Medline: 7479568]

27. Hacker E, Horsham C, Vagenas D, Jones L, Lowe J, Janda M. A mobile technology intervention with ultraviolet radiation dosimeters and smartphone apps for skin cancer prevention in young adults: randomized controlled trial. JMIR Mhealth Uhealth 2018 Nov 28;6(11):e199. [doi: 10.2196/mhealth.9854] [Medline: 30487115]

28. Buller DB, Berwick M, Lantz K, Buller MK, Shane J, Kane I, et al. Evaluation of immediate and 12-week effects of a smartphone sun-safety mobile application: a randomized clinical trial. JAMA Dermatol 2015 May;151(5):505-512 [FREE Full text] [doi: 10.1001/jamadermatol.2014.3894] [Medline: 25629819]

29. Horsham C, Antrobus J, Olsen CM, Ford H, Abernethy D, Hacker E. Testing wearable UV sensors to improve sun protection in young adults at an outdoor festival: field study. JMIR Mhealth Uhealth 2020 Sep 16;8(9):e21243 [FREE Full text] [doi: 10.2196/21243] [Medline: $\underline{32936083}$ ]

30. Bodekær M, Petersen B, Philipsen PA, Heydenreich J, Thieden E, Wulf HC. Sun exposure patterns of urban, suburban, and rural children: a dosimetry and diary study of 150 children. Photochem Photobiol Sci 2015;14(7):1282-1289. [doi: 10.1039/c5pp00052a] [Medline: 26066309]

31. Thieden E, Philipsen PA, Heydenreich J, Wulf HC. UV radiation exposure related to age, sex, occupation, and sun behavior based on time-stamped personal dosimeter readings. Arch Dermatol 2004 Feb 01;140(2):197-203. [doi: 10.1001/archderm.140.2.197] [Medline: 14967793]

32. Thieden E, Ågren M, Wulf H. Solar UVR exposures of indoor workers in a Working and a Holiday Period assessed by personal dosimeters and sun exposure diaries. Photodermatol Photoimmunol Photomed 2001;17(6):249-255. [doi: 10.1111/j.1600-0781.2001.170601.x] [Medline: 11722749]

33. Thieden E, Philipsen P, Wulf H. Ultraviolet radiation exposure pattern in winter compared with summer based on time-stamped personal dosimeter readings. Br J Dermatol 2006;154(1):133-138. [doi: 10.1111/j.1365-2133.2005.06961.x] [Medline: 16403106]

34. Baczynska KA, Khazova M, O'Hagan JB. Sun exposure of indoor workers in the UK - survey on the time spent outdoors. Photochem Photobiol Sci 2019 Jan 15;18(1):120-128. [doi: 10.1039/c8pp00425k] [Medline: 30357230]

35. Wu YP, Aspinwall LG, Conn BM, Stump T, Grahmann B, Leachman SA. A systematic review of interventions to improve adherence to melanoma preventive behaviors for individuals at elevated risk. Prev Med 2016 Dec;88:153-167 [FREE Full text] [doi: 10.1016/j.ypmed.2016.04.010] [Medline: 27090434]

36. Nahar VK, Ford MA, Brodell RT, Boyas JF, Jacks SK, Biviji-Sharma R, et al. Skin cancer prevention practices among malignant melanoma survivors: a systematic review. J Cancer Res Clin Oncol 2016 Jun;142(6):1273-1283. [doi: 10.1007/s00432-015-2086-z] [Medline: 26642962]

37. Finch L, Janda M, Loescher LJ, Hacker E. Can skin cancer prevention be improved through mobile technology interventions? A systematic review. Prev Med 2016 Sep;90:121-132. [doi: 10.1016/j.ypmed.2016.06.037] [Medline: 27374946]

38. Prospect lists - Consumer mailing list builder. Experian. 2018. URL: https://www.experian.com/small-business/mailing-lists [accessed 2019-02-16]

39. Shade UV sensor - Funded by the National Cancer Institute. Shade. URL: https://www.wearshade.com/ [accessed 2021-03-04]

40. UV index: Annual time series. National Weather Service - Climate Prediction Center. URL: $\underline{\text { https://www.cpc.ncep.noaa.gov/ }}$ products/stratosphere/uv index/uv annual.shtml [accessed 2021-02-04]

41. Harris PA, Taylor R, Thielke R, Payne J, Gonzalez N, Conde JG. Research electronic data capture (REDCap)--a metadata-driven methodology and workflow process for providing translational research informatics support. J Biomed Inform 2009 Apr;42(2):377-381 [FREE Full text] [doi: 10.1016/j.jbi.2008.08.010] [Medline: 18929686]

42. Glanz K, Yaroch AL, Dancel M, Saraiya M, Crane LA, Buller DB, et al. Measures of sun exposure and sun protection practices for behavioral and epidemiologic research. Arch Dermatol 2008 Feb;144(2):217-222. [doi: 10.1001/archdermatol.2007.46] [Medline: 18283179 ]

43. Brewer J, Mcquinn D, Lohse C, Hassani J. Tanning bed perception survey: A questionnaire-based study. J Clin Aesthet Dermatol 2015 Mar;8(3):23-27 [FREE Full text] [Medline: 25852811]

44. Aspinwall LG, Leaf SL, Dola ER, Kohlmann W, Leachman SA. CDKN2A/p16 Genetic test reporting improves early detection intentions and practices in high-risk melanoma families. Cancer Epidemiol Biomark Prev 2008 Jun 16;17(6):1510-1519. [doi: 10.1158/1055-9965.epi-08-0010] [Medline: $\underline{18559569]}$

45. Køster B, Søndergaard J, Nielsen JB, Allen M, Olsen A, Bentzen J. The validated sun exposure questionnaire: association of objective and subjective measures of sun exposure in a Danish population-based sample. Br J Dermatol 2017 Feb;176(2):446-456. [doi: 10.1111/bjd.14861] [Medline: 27412948]

46. Aspinwall L, Stump T, Taber J, Drummond D, Kohlmann W, Champine M. Genetic test reporting of CDKN2A provides informational and motivational benefits for managing melanoma risk. Transl Behav Med 2017;8(1):29-43. [doi: 10.1093/tbm/ibx011] [Medline: 29385581] 
47. Wu YP, Parsons BG, Aspinwall LG, Hay JL, Boucher KM, Caputo H, et al. Parent and child perspectives on perceived barriers to child sun protection and their association with sun protection strategies among children of melanoma survivors. Pediatr Dermatol 2019 Mar 20;36(3):317-323. [doi: 10.1111/pde.13796] [Medline: 30895676]

48. Lescano CM, Rodrigue JR. Skin cancer prevention behaviors among parents of young children. Child Health Care 1997 Apr;26(2):107-114. [doi: 10.1207/s15326888chc2602 4]

49. Nigg CR. Explaining adolescent exercise behavior change: A longitudinal application of the transtheoretical model. Ann Behav Med 2001 Feb;23(1):11-20. [doi: 10.1207/s15324796abm2301_3] [Medline: 11302350]

50. Prochaska JO, Velicer WF, Rossi JS, Goldstein MG, Marcus BH, Rakowski W, et al. Stages of change and decisional balance for 12 problem behaviors. Health Psychol 1994;13(1):39-46. [doi: 10.1037/0278-6133.13.1.39] [Medline: 8168470]

51. Rossi JS, Blais LM, Redding CA, Weinstock MA. Preventing skin cancer through behavior change. Implications for interventions. Dermatol Clin 1995 Jul;13(3):613-622. [doi: 10.1016/S0733-8635(18)30067-6] [Medline: 7554509]

52. Tribby CP, Julian AK, Oh AY, Perna FM, Berrigan D. Associations between ultraviolet radiation, tree cover and adolescent sunburns. Int J Health Geogr 2020 Dec 14;19(1):59. [doi: 10.1186/s12942-020-00253-x] [Medline: 33317555]

53. Holmbeck G. Post-hoc probing of significant moderational and mediational effects in studies of pediatric populations. $\mathrm{J}$ Pediatr Psychol 2002;27(1):87-96. [doi: 10.1093/jpepsy/27.1.87] [Medline: 11726683 ]

\title{
Abbreviations \\ REDCap: Research Electronic Data Capture \\ UVR: UV radiation
}

\author{
Edited by L Buis; submitted 22.04.21; peer-reviewed by T Stump, J Robinson; comments to author 25.05.21; revised version received \\ 23.06.21; accepted 03.08.21; published 28.09.21 \\ Please cite as: \\ Parsons BG, Nagelhout ES, Wankier AP, Hu N, Lensink R, Zhu A, Nottingham K, Grossman D, Jensen JD, Wu YP \\ Reactivity to UV Radiation Exposure Monitoring Using Personal Exposure Devices for Skin Cancer Prevention: Longitudinal \\ Observational Study \\ JMIR Mhealth Uhealth 2021;9(9):e29694 \\ URL: https://mhealth.jmir.org/2021/9/e29694 \\ doi: $10.2196 / 29694$ \\ PMID:
}

(CBridget G Parsons, Elizabeth S Nagelhout, Ali P Wankier, Nan Hu, Riley Lensink, Angela Zhu, Katy Nottingham, Douglas Grossman, Jakob D Jensen, Yelena P Wu. Originally published in JMIR mHealth and uHealth (https://mhealth.jmir.org), 28.09.2021. This is an open-access article distributed under the terms of the Creative Commons Attribution License (https://creativecommons.org/licenses/by/4.0/), which permits unrestricted use, distribution, and reproduction in any medium, provided the original work, first published in JMIR mHealth and uHealth, is properly cited. The complete bibliographic information, a link to the original publication on https://mhealth.jmir.org/, as well as this copyright and license information must be included. 